\title{
COMMISSION 36: THEORY OF STELLAR ATMOSPHERES
} (THEORIE DES ATMOSPHERES STELLAIRES)

\author{
PRESIDENT: Dainis Dravins \\ VICE-PRESIDENT: Monique Spite \\ ORGANIZING COMMITTEE: T. Ayres, B. Barbuy, L. Cram, D. Kiselman, \\ J. Landstreet, R. Pallavicini, H. Saio, C. Sneden, K. Stępień \& K.Werner
}

\section{Introduction}

This report is an overview of research during the past triennium. Since the breadth of the field makes it unfeasible to highlight more than a few small samples of it, it was decided to shorten this report as compared to that of earlier triennia. It lists areas of current research, and provides web links for obtaining further information:

\section{Primary Research Areas 1999-2002}

\subsection{Physical Processes}

General properties Improved models available to the community; Grids of synthetic fluxes and spectra; Calibrating parameterized models through physical modeling; Calibrating abundance determinations by filter photometry or low-resolution spectroscopy

Stationary processes within stellar atmospheres Convection (granulation) in surface layers, and its effects upon emergent spectra; Interplay between convection and non-radial pulsation; Scales of surface convection in stars in different stages of evolution; Hydrodynamic simulations of entire stellar volumes

Transient processes Shocks in pulsating stars; Radiative cooling of shocked gas; Emission lines as shock-wave diagnostics; Particle acceleration during flares; Interaction of jets with interstellar medium; Episodic outflows and star-disk interaction

Magnetic phenomena Magnetic structures in single and binary stars; Dynamo generation of magnetic fields by convection; Effects by magnetic fields on convective structures; Magnetic cycles at varying activity levels; Polarized radiation, gyrosynchrotron and X-ray emission; Interpreting Zeeman-Doppler images of stellar surfaces

Radiative transfer and emergent stellar spectra Effects on atmospheric structure by deviations from local thermodynamic equilibrium (non-LTE); Multidimensional radiative transfer; Radiative hydrodynamics; Origin and transfer of polarized light

Spectral lines, and their formation Line formation in convective atmospheres; Wavelength shifts as signatures of convection; Spectra of rotating stars viewed pole-on and equator-on; Non-LTE effects in permitted and forbidden lines; Atomic and quantum processes affecting spectral lines; Databases for spectral lines; Atlases of synthetic spectra 
Forbidden lines 8 maser emission Molecules in atmospheres of cool giant stars; Effects of fluorescence; Permitted and forbidden lines from shocked atmospheres of pulsating giants; Maser and laser emission from stellar envelopes

Chemical abundances Abundance anomalies; Hydrodynamical models of metal-poor stars; Depletion of light elements through atmospheric motions; Pollution of atmospheres by interstellar dust; r- and s-process elements; Chemical stratification in stable atmospheres; Coronal versus photospheric abundances; Chemical inhomogeneities and pulsation

Molecules Chromospheric structures and temperature inhomogeneities; Cool molecular constituents in warm stars; Molecular spheres around red giants; Molecular opacities, and non-LTE effects; Role of molecular hydrogen

\subsection{Stellar Structures}

Structures across stellar disks Doppler mapping of starspots; Radii and oblateness at different wavelengths for giant stars; Gravitational microlensing to test model atmospheres; Interaction between rotation and pulsation; Doppler tomography of stellar envelopes

Stellar coronae Coronal heating mechanisms (quiescent and flaring); Effects of age and chemical abundance; Multicomponent structure; Coronae in also low-mass stars and brown dwarfs; Diagnostics through $\mathrm{X}$-ray spectroscopy and radio emission

Stellar winds and mass loss Dynamic outer atmospheres; Multi-component radiation- and dust-driven winds; Mass loss from pulsating giants; Effects of mass flows on the ionization structure; Coronal mass ejections; Instabilities in hot-star winds; X-ray emission

Dust, grains, and shells Formation of stellar dust shells; Grains in the atmospheres of red giants, and in T Tauri stars

\subsection{Different Classes of Objects}

Pulsating stars and asteroseismology Classically variable stars, and 'ordinary' solar-type ones; Inverting observed pressure-mode frequencies into atmospheric structure; Mass-loss mechanisms in pulsating stars; Effects of rapid rotation on pulsation

Binary stars Atmospheric structure and magnetic dynamos in common-envelope binaries; Role of binarity on mass loss; Tidal effects; Non-LTE effects by illumination from the component; Reflection effects in close binaries; Colliding stellar winds

New classes of very cool stars Dust, clouds, weather, and chemistry in brown dwarfs; Cloud clearings and hot-spots; Magnetic activity; The effective temperature scale; Molecular line and continuum opacities; Transition between extrasolar giant planets and ultracool brown dwarfs

White dwarfs and neutron stars Radiative transfer in magnetized white-dwarf atmospheres; Stokes-parameter imaging of white dwarfs; Molecular opacities in white dwarfs; Atmospheres and spectra of neutron stars; Effects of vacuum polarization and accretion around magnetized neutron stars

Special objects Central stars of planetary nebulae; Population III stars of extremely low metallicity; Protostars; Accretion disks and coronal activity in young stars

Interaction with exoplanets Effects of planets on the atmospheres of evolved red giants; Characteristics of stars hosting exoplanets 


\subsection{Development of Techniques}

Computational techniques Parallel [super]computing to simulate convective surface regions, and throughout complete stars; Neural networks and machine-learning algorithms; Preparing for the widely distributed network of computational tools and shared databases being developed for the forthcoming computing infrastructure GRID

\subsection{Applications of Stellar Atmospheres}

Besides their study per se, stars are being used as probes for other astrophysical problems:

Exoplanets Variable wavelength shifts in stellar spectra serve as diagnostics for radialvelocity variations induced by orbiting exoplanets. Atmospheric modeling can indicate which spectral features are suitable as such probes, and which should be avoided due to their sensitivity to intrinsic stellar activity.

Chemical evolution in the Galaxy How accurately observations of stellar spectral features can be transformed into actual chemical abundances depends sensitively on the sophistication of the stellar model atmospheres.

Kinematics of the Galaxy Planned space missions intend to measure radial velocities for huge numbers of stars. Model atmospheres are used to identify suitable spectral features for such measurements in different classes of stars.

Galaxies and cosmology Stars are the main observable component of galaxies, and population synthesis for galaxies utilize model atmospheres to interpret observations; Cosmological origin of the lowest-metallicity stars.

\section{Web Links for Further Information}

The widespread use of the world-wide-web in most parts of the astronomical world has made it a primary source for seeking introductions to the field. Unfortunately, web addresses have had a tendency to be only short-lived, limiting the value of their publication. The following collection of links, valid at the time of writing, provides introductions and overviews of several significant subfields of the physics of stellar atmospheres:

\subsection{Calculating Atmospheric Models and Spectra}

ATLAS, SYNTHE, and other model grids http://kurucz.harvard.edu

CCP7 - Collaborative Computational Project http://ccp7.dur.ac.uk/

CLOUDY - photoionization simulations http://www.pa.uky.edu/ gary/cloudy/

MULTI - non-LTE radiative transfer http://www.astro.uio.no/ matsc/mul22/

PANDORA - atmospheric models and spectra http://cfa-www.harvard.edu/ rloeser/pandora.html

PHOENIX - stellar and planetary atmosphere code http://phoenix.physast.uga.edu/

STARLINK - theory and modeling resources http://www.astro.gla.ac.uk/users/norman/star/sc13/sc13.htx/

Synthetic spectra overview http://www.am.ub.es/ carrasco/models/synthetic.html 
TLUSTY - model atmospheres http://tlusty.gsfc.nasa.gov/

\subsection{Research Groups}

Munich: Hot stars

http://www.usm.uni-muenchen.de/people/adi/adi.html

Potsdam: Stellar convection http://www.aip.de/groups/sternphysik/stp/convect_neu.html

Tübingen: Stellar atmospheres http://astro.uni-tuebingen.de/groups/stellar/

Vienna: Stellar atmospheres and pulsating stars http://ams.astro.univie.ac.at/main.php

\subsection{Individual Researchers}

M. Asplund: Stellar convection \& line formation http://www.mso.anu.edu.au/ martin/

G. Basri: Brown dwarfs http://astro.berkeley.edu/ basri/bdwarfs/

A. Collier Cameron: Starspots and magnetic fields on cool stars http://star-www.st-and.ac.uk/ acc4/coolpages/imaging.html

J.F. Donati: Magnetic fields of non degenerate stars http://webast.ast.obs-mip.fr/people/donati/field.html

D. Dravins: Stellar surface structure http://www.astro.lu.se/ dainis/HTML/GRANUL.html

D.F. Gray: Stellar rotation, magnetic cycles, velocity fields http://phobos.astro.uwo.ca/ dfgray/

M. Jardine: Stellar coronal structure http://capella.st-and.ac.uk/ mmj/Welcome_research.html

S. Jeffery: Stellar model grids, hot stars http://star.arm.ac.uk/ csj/

J.L. Linsky: Cool stars, stellar chromospheres and coronae http://jilawww.colorado.edu/ jlinsky/

D. Montes et al.: Libraries of stellar spectra http://www.ucm.es/info/Astrof/spectra.html

R.J. Rutten: Lecture notes: Radiative transfer in stellar atmospheres http://www.fys.ruu.nl/ rutten/node20.html

P. Stee: Be-star atmospheres and circumstellar envelopes http://www.obs-nice.fr/stee/Bemodel.html http://www.obs-nice.fr/stee/simugb.html

R.F. Stein: Convection simulations \& radiation hydrodynamics http://www.pa.msu.edu/ steinr/research.html $\not$ research

Dainis Dravins

President of the Commission 